\title{
Predictors of toxicity in treating patients with neuroblastoma by radiolabeled metaiodobenzylguanidine
}

\author{
James C. Sisson ${ }^{1}$, Brahm Shapiro', Raymond J. Hutchinson², James E. Carey', Kenneth R. Zasadny', \\ Shirley A. Zempel ${ }^{1}$, Daniel P. Normolle ${ }^{3}$ \\ 1 Department of Internal Medicine, Division of Nuclear Medicine, University of Michigan Medical Center, \\ 1500 E. Medical Center Drive, Ann Arbor, MI 48109-0028, USA \\ ${ }^{2}$ Department of Pediatrics, Division of Hematology and Oncology, University of Michigan Medical Center, \\ 1500 E. Medical Center Drive, Ann Arbor, MI 48109-0028, USA \\ ${ }^{3}$ Department of Biostatistics, School of Public Health, University of Michigan Medical Center, 1500 E. Medical Center Drive, \\ Ann Arbor, MI 48109-0028, USA
}

Received 10 July and in revised form 9 September 1993

\begin{abstract}
We searched for methods that would enable prescriptions of the maximum tolerable doses of iodine131 metaiodobenzylguanidine (MIBG) and iodine- 125 MIBG in the treatment of patients with neuroblastoma. We correlated doses, defined in different ways, with subsequent platelet levels in treated patients to determine accurate predictors of the most frequent toxicity, thrombocytopenia. Nine patients with neuroblastoma were given ${ }^{131} \mathrm{I}-\mathrm{MIBG}(4.9-8.1 \mathrm{GBq}$ or $132-220 \mathrm{mCi})$ and ten were given ${ }^{125}$ I-MIBG $(8.3-30.0 \mathrm{GBq}$ or 224 $809 \mathrm{mCi}$ ) as initial treatments. These therapies were sufficiently varied that correlations could be made between indices of the doses and the subsequent toxicity as reflected in circulating platelet levels. Predictors of toxicity were: whole-body absorbed dose of radiation (cGy) calculated from pretherapy tracer doses of ${ }^{131} \mathrm{I}-$ MIBG; $\mathrm{GBq} / \mathrm{kg}$ of body weight; and $\mathrm{GBq} / \mathrm{m}^{2}$ of body surface area. Toxicity was recorded as the nadir of the platelet level and platelet/pretherapeutic level (platelet ratio). For treatments with ${ }^{131} \mathrm{I}-\mathrm{MIBG}$, the highest correlation was obtained between cGy and the $\log _{10}$-transformed platelet ratio $(r=-0.86)$, but comparison of $\mathrm{GBq} / \mathrm{m}^{2}$ and the platelet nadir $(r=-0.76)$ or the platelet ratio $(r=-0.74)$ or the $\log _{10}{ }^{-}$transformed platelet ratio $(r=-0.73)$ gave comparable and statistically significant results. For treatments with ${ }^{125} \mathrm{I}-\mathrm{MIBG}$, significant correlations were obtained between $\mathrm{GBq} / \mathrm{m}^{2}$ and the platelet ratio $(r=-0.81)$ or $\mathrm{GBq} / \mathrm{kg}$ and the $\log _{10^{-}}$-transformed platelet ratio; the correlation between $\mathrm{CGy}$ and any toxicity index was low. Per administered GBq, ${ }^{131}$ I-MIBG was 2.6 times more potent than ${ }^{125} \mathrm{I}-\mathrm{MIBG}$ in causing a platelet ratio of 0.1 . Thus, in predicting toxicity, therapeutic doses of ${ }^{131} \mathrm{I}-\mathrm{MIBG}$ expressed as $\mathrm{GBq} / \mathrm{m}^{2}$ performed satisfactorily and almost as well as whole-body
\end{abstract}

Correspondence to: J.C. Sisson
cGy, and treatment doses of ${ }^{125} \mathrm{I}-\mathrm{MIBG}$ expressed as $\mathrm{GBq} / \mathrm{m}^{2}$ or $\mathrm{GBq} / \mathrm{kg}$ performed satisfactorily and much better than whole-body cGy.

Key words: Metaiodobenzylguanidine - Therapy - Toxicity - Thrombocytopenia - Dosimetry

Eur J Nucl Med (1994) 21:46-52

\section{Introduction}

Radiolabeled metaiodobenzylguanidine (MIBG) has been introduced as therapy for patients with advanced neuroblastoma because other treatments have failed to effect cures and because the concept of delivering radiation selectively to the tumors - via the sequestration of MIBG - is compelling. Therapies with ${ }^{131} \mathrm{I}-\mathrm{MIBG}$ appeared to cause regressions of tumors in about onefourth of the treated patients; there were a few complete remissions, but no cures [1-11]. To attack the micrometastases that may be the source of recurrences following ${ }^{131}$ I-MIBG treatments, ${ }^{125}$ I-MIBG, which can impart more confined radiation energy within small neoplastic deposits, was given as a therapeutic agent in a phase I/II trial $[12,13]$.

If the radiolabeled MIBGs are to have a role in the treatment of neuroblastoma, optimum effects are likely to occur only when the agents are given in large doses that result in some toxicity to normal tissues. On the other hand, current practice is not to produce such severe toxicity that the most vulnerable organ, the bone marrow, fails to maintain function. Within this guideline, thrombocytopenia, the most sensitive expression of radiation injury from the radiopharmaceuticals $[1,6$, 12-14], particularly must not become life threatening. 
Therefore, a reliable predictor of bone marrow toxicity is necessary if aggressive therapies with ${ }^{131} \mathrm{I}-\mathrm{MIBG}$ and/or ${ }^{125}$ I-MIBG are to be pursued. We report here on our analyses of the toxic consequences of treatments, at varied administered doses of ${ }^{131} \mathrm{I}-\mathrm{MIBG}$ and ${ }^{125} \mathrm{I}-$ MIBG, and derive indices that predict thrombocytopenia.

\section{Materials and methods}

Patients. Data on six of the patients treated with ${ }^{131}$ I-MIBG have been reported in part elsewhere [14]. Three additional patients were treated with ${ }^{131}$ I-MIBG. They were recruited for ${ }^{125}$ I-MIBG treatment; however, because ${ }^{125} \mathrm{I}$ was not available in sufficient quantities for synthesis of the radiopharmaceutical at the time of treatment, these patients were given ${ }^{131}$ I-MIBG instead. Clinical information on seven of the patients has been included in other publications [7, 15].

Ten subjects were recruited for ${ }^{125}$ I-MIBG treatment from pediatric oncologists around the United States. Entry criteria for treatment with ${ }^{125}$ I-MIBG were as described before, but also included patients with progressive disease [12]. Preliminary data on the first seven patients in the ${ }^{125}$ I-MIBG treated group have also been reported previously $[12,13]$. No patient had received prior therapy with a radiolabeled MIBG. The patients exhibited little (less than $25 \%$ ) or no bone marrow involvement by the neuroblastoma. Levels of peripheral blood elements including platelets were measured by standard automated techniques.

This investigation was approved by Internal Review Board at the University of Michigan Medical Center.

Radiopharmaceuticals. Syntheses of ${ }^{131}$ I-MIBG and ${ }^{125}$ I-MIBG were carried out as previously described [12, 16, 17]. For diagnostic doses ${ }^{131} \mathrm{I}-\mathrm{MIBG}$ was prepared at $0.12-0.13 \mathrm{GBq} / \mathrm{mg}$, mean $0.12 \mathrm{GBq} / \mathrm{mg}(1.1 \mathrm{Ci} / \mathrm{mmol})$. For therapies the specific activities were $1.0-1.94 \mathrm{GBq} / \mathrm{mg}(9.1-17.4 \mathrm{Ci} / \mathrm{mmol})$ for ${ }^{131} \mathrm{I}$ MIBG and $40.0-63.8 \mathrm{GBq} / \mathrm{mg}(13.3-21.3 \mathrm{Ci} / \mathrm{mmol})$ for ${ }^{125} \mathrm{I}-$ MIBG. These values can be calculated for individual patients from the data in Table 1 .

Dosimetry. Methods of dosimetry using ${ }^{131}$ I-MIBG have also been previously described $[12,14]$. Briefly, ${ }^{131}$ I-MIBG, 0.063 $\mathrm{GBq} / \mathrm{m}^{2}$ of body surface area, was infused over $90 \mathrm{~min}$. Wholebody counting for residual radioactivity using a scintillation probe at $2 \mathrm{~m}$ was performed shortly after completion of the infusion to give a $100 \%$ value and again after voiding urine at $3 \mathrm{~h}$. The geometric means of anterior and posterior counts were used in calculations. Subsequent measurements were made daily from 1 through 6 or 7 days. Blood samples were obtained immediately following the infusion, at $3 \mathrm{~h}$ and then daily at the time of measurements of whole-body retention. Whole blood specimens were counted in a well counter.

Table 1. Data on individual therapies

\begin{tabular}{|c|c|c|c|c|c|c|c|c|c|c|}
\hline \multirow[t]{2}{*}{ Patient } & \multirow{2}{*}{$\begin{array}{l}\text { Weight } \\
(\mathrm{kg})\end{array}$} & \multirow{2}{*}{$\begin{array}{l}\text { Surface } \\
\text { area } \\
\left(\mathrm{m}^{2}\right)\end{array}$} & \multicolumn{4}{|c|}{ MIBG administered doses } & \multicolumn{2}{|c|}{ Absorbed radiation } & \multicolumn{2}{|c|}{ Circulating platelets } \\
\hline & & & $\mathrm{mg}^{\mathrm{a}}$ & $\mathrm{GBq}$ & $\mathrm{GBq} / \mathrm{kg}$ & $\mathrm{GBq} / \mathrm{m}^{2}$ & $\begin{array}{l}\text { Whole } \\
\text { body } \\
\text { (cGy) }\end{array}$ & $\begin{array}{l}\text { Blood } \\
\text { (cGy) }\end{array}$ & $\begin{array}{l}\text { Nadir } \\
\text { (thousands) }\end{array}$ & Ratio $^{b}$ \\
\hline
\end{tabular}

A. ${ }^{131} \mathrm{I}-\mathrm{MIBG}$ Therapies

$\begin{array}{lll}1 & 14 & 0.59 \\ 2 & 19.8 & 0.77 \\ 3 & 19.8 & 0.83 \\ 4 & 11.4 & 0.51 \\ 5 & 20 & 0.82 \\ 6 & 56.6 & 1.61 \\ 7 & 19.8 & 0.83 \\ 8 & 15.5 & 0.70 \\ 9 & 18.5 & 0.75\end{array}$

$\begin{array}{ll}5.1 & 5.14 \\ 4.3 & 7.51 \\ 5.1 & 7.40 \\ 3.3 & 4.88 \\ 4.7 & 7.84 \\ 4.2 & 8.14 \\ 3.4 & 6.88 \\ 4.1 & 6.48 \\ 4.9 & 7.40\end{array}$

0.366
0.381
0.374
0.429
0.392
0.144
0.347
0.422
0.400

8.66
19.8
8.95
9.58
9.51
5.07
8.33
9.25
9.92

231
183
180
170
132
103
179
201
305

$\begin{array}{rr}110 & 89 \\ 88 & 57 \\ 76 & 173 \\ 84 & 131 \\ - & 116 \\ 59 & 342 \\ - & 233 \\ - & 78 \\ - & 10\end{array}$

0.30

0.26

$$
0.45
$$$$
0.45
$$$$
0.29
$$$$
0.97
$$$$
0.73
$$$$
0.27
$$$$
0.11
$$

B. ${ }^{125} \mathrm{I}-\mathrm{MIBG}$ Therapies

\begin{tabular}{lllr}
1 & 14.9 & 0.69 & 6.1 \\
$2^{\mathrm{c}}$ & 13.8 & 0.60 & 5.8 \\
3 & 15.7 & 0.64 & 8.9 \\
$4^{\mathrm{d}}$ & 21.2 & 0.86 & 8.9 \\
5 & 15.7 & 0.64 & 8.9 \\
$6^{\mathrm{d}}$ & 18.1 & 0.75 & 8.2 \\
$7^{\mathrm{d}}$ & 42.6 & 1.38 & 14.6 \\
8 & 15.5 & 0.68 & 7.6 \\
$9^{\mathrm{c}}$ & 18.2 & 0.69 & 5.9 \\
10 & 26.8 & 1.02 & 17.7 \\
\hline
\end{tabular}

$\begin{array}{rcllrr}6.1 & 13.7 & 0.899 & 20.0 & 105 & 69 \\ 5.8 & 9.66 & 0.699 & 16.1 & 83 & 55 \\ 8.9 & 15.1 & 0.958 & 23.5 & 104 & 73 \\ 8.9 & 19.7 & 0.929 & 23.0 & 133 & 89 \\ 8.9 & 20.0 & 1.27 & 31.5 & 118 & 82 \\ 8.2 & 14.8 & 0.821 & 19.9 & 105 & 69 \\ 14.6 & 29.9 & 0.703 & 21.8 & 84 & 61 \\ 7.6 & 16.0 & 1.03 & 23.3 & 120 & \\ 5.9 & 8.29 & 0.459 & 12.0 & 119 & 80.5 \\ 17.7 & 30.1 & 1.12 & 29.5 & 120 & 66\end{array}$

$\begin{array}{rrl}69 & 16 & 0.11 \\ 55 & 176 & 0.49 \\ 73 & 207 & 0.50 \\ 89 & 58 & 0.25 \\ 82 & 7 & 0.017 \\ 69 & 43 & 0.23 \\ 61 & 66 & 0.35 \\ - & 27 & 0.17 \\ 80 & 204 & 0.47 \\ 66 & 6 & 0.022\end{array}$

${ }^{a}$ In ${ }^{125}$ I-MIBG therapies, $0.3 \mathrm{mg}$ of ${ }^{131} \mathrm{I}$-MIBG is included

${ }^{b}$ Platelet ratio is nadir level/level at outset of therapy

c One kidney

${ }^{\mathrm{d}}$ Had bone marrow transplants 
Absorbed dose was calculated following the MIRD formalism [18]. The equation for calculating the mean absorbed dose was:

$\bar{D}=\frac{\tilde{A}}{m} \Sigma \Delta_{\mathrm{i}} \Phi_{\mathrm{i}}$

where, $\bar{D}$, in Gy, was the mean absorbed dose; $\tilde{A}$, in Bq-s, was the cumulated activity; $\Delta_{\mathrm{i}}$, in Gy-kg per Bq-s, was the mean energy emitted per unit cumulated activity for the $\mathrm{i}$-th type radiation; $\theta_{\mathrm{i}}$ was the absorbed fraction for the $\mathrm{i}$-th type of radiation; and $m$, in $\mathrm{kg}$, was the mass of the target organ.

The cumulated activity was obtained by integration under the patient's whole-body or blood time-activity curves. For time following the last measured data point, an effective half-life was determined from the slope (obtained by linear regression analysis) of the last three measured data points. The area under this "tail" was obtained using the equation:

$\tilde{A}=1.443 \times A_{n} \times T_{\text {eff }}$,

where, $A_{\mathrm{n}}$, in $\mathrm{Bq}$, was the activity of the last data point measured at time $n$; and $T_{\text {eff }}$, in seconds, was the calculated effective half-life.

The mean energy emitted per unit cumulated activity was obtained from ICRU Report 32 [19]. The absorbed fractions for beta particles and other nonpenetrating radiations were assumed to be one. For the whole body, the absorbed fractions for gamma and other penetrating radiation were obtained from tables in MIRD Pamphlet No. 3 [20] assuming uniform distribution of the activity in an ellipsoid having the same mass as the patient. For blood, the absorbed fractions for gamma and other penetrating radiation were assumed to be equal to the absorbed fraction for the whole body. The time-activity curves for blood showed a more rapid disappearance of radiolabeled MIBG than those for the whole body. Thus, absorbed radiation doses to blood were always substantially lower than those to the body.

Treatments. Therapeutic doses were also infused over $90 \mathrm{~min}$. Therapeutic doses of ${ }^{125} \mathrm{I}-\mathrm{MIBG}$ included $0.037 \mathrm{GBq}$ of ${ }^{131} \mathrm{I}$ MIBG to facilitate imaging of the distribution of the radiopharmaceutical. Whole-body retentions of the treatment doses were measured by an ion chamber at $1 \mathrm{~m}$ in the room of each patient. These measurements were almost entirely of radiations from ${ }^{125} \mathrm{I}$. The times of measurement were the same as for the pretherapy dosimetry, but the patients frequently were discharged 4 days after treatment and, in two instances, data were collected for only 3 days.

Of those receiving ${ }^{131}$ I-MIBG, the first six patients had received ${ }^{131}$ I-MIBG in doses that varied according to the accumulating experience in what was then a new therapy [4]. As noted above, three additional patients were given $6.48-7.40 \mathrm{GBq}$ of ${ }^{131}$ I-MIBG, doses that had produced some, but not marked, declines in circulating platelets of the previous six subjects (Table 1).

In the phase I/II trial of therapy with ${ }^{125} \mathrm{I}-\mathrm{MIBG}$, a protocol was designed in which doses were to be escalated to find the maximum tolerable dose of this radiopharmaceutical, one that produced marked but tolerable and reversible toxicity. Initially, we believed that absorbed doses of whole-body radiation would best predict toxicity. Doses in the escalation protocol were to be 75-124 cGy, 124-175 cGy, etc., each for groups of three patients. However, it soon became apparent that some doses in the 125 cGy range produced marked thrombocytopenia, but others did not, and whole-body radiation doses proved to be inaccurate predictors of toxicity. Subsequent doses were then aimed at the 120 130 cGy level to gain added information (Table 1). However, for two patients $(\# 6, \# 7)$ the syntheses of ${ }^{125}$ I-MIBG produced doses for administration that would impart somewhat less than this target for whole-body radiation. Nevertheless, since patients were prepared for, and anticipated treatment, we decided to proceed with the less than prescribed doses in these instances.

Because previous experience had shown that thrombocytopenia was the earliest sign of toxicity $[1,6,12,14]$, we adopted as the indices of toxicity the relative declines and the absolute levels of circulating platelets. These data are also included in Table 1.

Data analyses. The two toxicity indices, lowest post-therapeutic level of circulating platelets (nadir) and relative change in platelets (platelet ratio, calculated as nadir/pretherapeutic platelet count), were compared to three predictive indices, whole-body absorbed dose in $c G y$, administered dose in $G B q / \mathrm{kg}$ of body weight, and administered dose in $G B q / \mathrm{m}^{2}$ of body surface area. The logarithmic (base 10 , hence $\log _{10}$ ) transformation was applied to the platelet ratio to produce a more linear relationship with the predictive indices [21]. The logit transformation $\{\operatorname{logit}(p)=$ $\log _{10}[p /(1-p]\}$, which is known to improve the linearity of relationships involving proportions [21], was also applied to the platelet ratio, but the results were virtually identical to those involving the $\log _{10}$ transformation and are not reported here.

The quality of the comparisons between predictive and toxicity indices was assessed by the magnitude of the correlations and by visual examination of bivariate plots for leverage points (observations far removed from the others along the horizontal axis which would artificially increase the size of the correlation coefficient). If an observation was found to be a leverage point, the analysis was repeated with that value deleted, resulting in a conservative estimate of the correlation coefficient. In all the analyses, only the values of whole-body dose measured in $\mathrm{GBq} / \mathrm{kg}$ and in $\mathrm{GBq} / \mathrm{m}^{2}$ for patient \#6 treated with ${ }^{131}$ I-MIBG were found to exert undue influence on the regression (see Fig. 2). Patient \#6 was an adult who weighed $56.6 \mathrm{~kg}$ and received $8.14 \mathrm{GBq}$, so that his predictive indices were relatively low, and he exhibited little or no toxicity. This patient's values were deleted from analyses involving $\mathrm{GBq} / \mathrm{kg}$ and $\mathrm{GBq} / \mathrm{m}^{2}$.

All statistical calculations were performed using the Statistical Analysis System (SAS Institute, Cary, NC). $p$-Values were determined by dividing the value of the regression coefficients by their standard errors and comparing the results to a $t$-distribution. If the correlation between a predictive index and a toxicity index was significant, the estimated toxic dose (in units of the predictive index) was calculated. For this calculation, toxicity was assumed to occur if the count declined beneath 20,000 platelets/ $\mu \mathrm{l}$ or if the platelet ratio declined beneath 0.1 . The estimated toxic dose was derived by inverting the regression equation:

toxicity index $=a+b \times$ predictive index

and inserting the given toxicity level to obtain:

toxic dose $=($ toxicity level $-a) / b$.

Correlations were also made between the absorbed radiation dose in blood and the indices of toxicity. However, data for the blood doses were not obtained for four patients treated with ${ }^{131} \mathrm{I}$ MIBG and for one patient treated with ${ }^{125}$ I-MIBG.

\section{Results}

\section{Treatment with ${ }^{131} I-M I B G$}

The highest correlations between predictive and toxicity indices were produced for the predictive indices 
Table 2. Correlations of predictive indices with toxicity indices

\begin{tabular}{|c|c|c|c|c|c|c|}
\hline \multirow[t]{7}{*}{${ }^{131} I$} & \multirow[t]{3}{*}{$\mathrm{GBq} / \mathrm{kg}$} & $r$ & -0.48 & -0.32 & -0.47 & -0.38 \\
\hline & & $P$ & 0.23 & 0.44 & 0.24 & 0.35 \\
\hline & & Toxicity & - & - & - & - \\
\hline & $\mathrm{GBq} / \mathrm{m}^{2}$ & $r$ & -0.76 & -0.68 & -0.74 & -0.72 \\
\hline & \multirow[t]{3}{*}{ cGy } & $r$ & -0.74 & -0.86 & -0.69 & -0.79 \\
\hline & & $P$ & 0.02 & 0.003 & 0.04 & 0.01 \\
\hline & & Toxicity & 277 & 292 & 290 & 270 \\
\hline \multirow[t]{6}{*}{${ }^{125} \mathrm{I}$} & $\mathrm{GBq} / \mathrm{kg}$ & $r$ & -0.66 & -0.78 & -0.76 & -0.80 \\
\hline & \multirow{2}{*}{$\mathrm{GBq} / \mathrm{m}^{2}$} & $P$ & 0.04 & 0.01 & 0.02 & 0.005 \\
\hline & & Toxicity & 773 & 724 & 779 & 675 \\
\hline & \multirow[t]{3}{*}{ cGy } & $r$ & -0.31 & -0.37 & -0.46 & -0.41 \\
\hline & & $P$ & 0.38 & 0.29 & 0.18 & 0.24 \\
\hline & & Toxicity & - & - & - & - \\
\hline
\end{tabular}

Correlation coefficient $(r)$, statistical significance of correlations $(P)$, and estimate of toxic dose for different predictive and toxicity indices. Toxicity was assumed to occur at a platelet count $\leq$ 20,000 platelets $/ \mu$ or at a nadir/pretreatment ratio $\leq 0.1$. Estimates

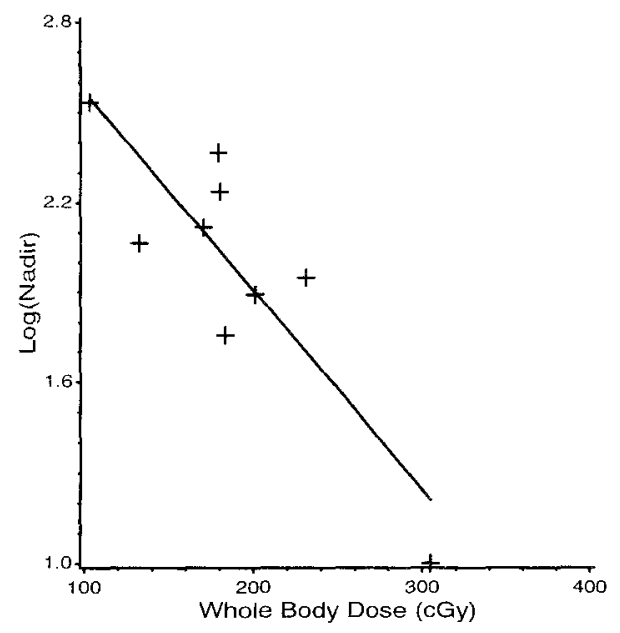

Fig. 1. $\log _{10}$-transformed nadir of platelets in thousands versus dose of ${ }^{131} \mathrm{I}-\mathrm{MIBG}$ in cGy. The equation for the plotted regression line is $\log _{10}$ (nadir) $=3.23-0.0066 \times \mathrm{cGy} ; r=-0.85, P=0.003$

$\mathrm{GBq} / \mathrm{m}^{2}$ and cGy (Table 2). The value of the $\log _{10}$ transformation on these indices was difficult to assess, but the indices that predicted toxicity, $10.2-10.8 \mathrm{GBq} / \mathrm{m}^{2}$ and 277-292 cGy, were consistent irrespective of the use of the transformation.

The largest correlation between predictive and toxicity indices for ${ }^{131}$ I-MIBG was that between wholebody radiation (in cGy) and $\log _{10}$-transformed platelet of toxicity were not determined when $P$-value $>0.10$. Sample size for all ${ }^{125}$ I-MIBG values is $n=10$. Sample size for all ${ }^{131} \mathrm{I}$ MIBG values is $n=9$, except for values related to $\mathrm{GBq} / \mathrm{kg}$ and $\mathrm{GBq} / \mathrm{m}^{2}$, where sample size is $n=8$ (see Materials and methods)

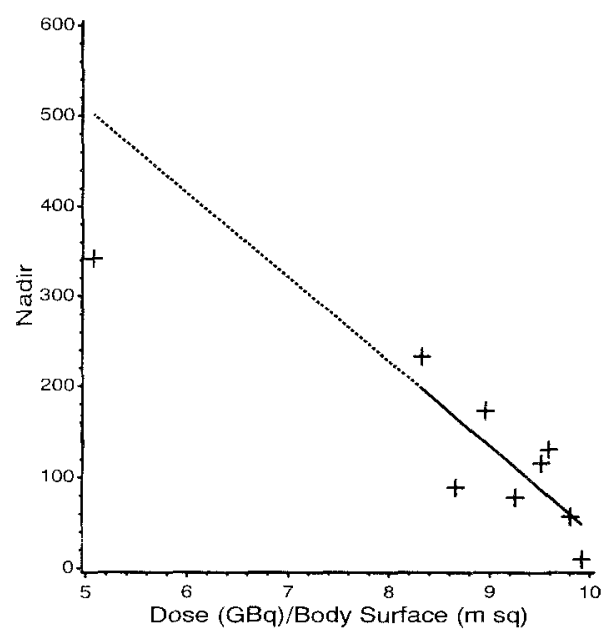

Fig. 2. Platelet nadir in thousands versus dose of ${ }^{13 !} \mathrm{I}-\mathrm{MIBG}$ in $\mathrm{GBq} / \mathrm{m}^{2}$. The equation for the plotted regression line is nadir $=974$ $-93.2 \times \mathrm{GBq} / \mathrm{m}^{2} ; r=-0.76, P=0.03$. Patient $\# 6\left(\right.$ dose $\left.=5.1 \mathrm{GBq} / \mathrm{m}^{2}\right)$ was not included in the calculation of the regression line

nadir, $r=-0.85(n=9, P=0.003$ ) (Fig. 1). If toxicity is defined as a platelet count of $20000 / \mu$ l or less (or 20 for simplification), this regression is: $\log _{10}$ (nadir) $=3.23$ $-0.0066 \times$ dose in $\mathrm{cGy}$; and $\log _{10}(20)=1.3$ then predicts toxicity at 292 cGy.

Good results were also achieved by comparing the nadir to the predictive index $\mathrm{GBq} / \mathrm{m}^{2}(r=-0.76, P=0.03)$ (Fig. 2). Toxicity was predicted at $10.2 \mathrm{GBq} / \mathrm{m}^{2}$, a value 


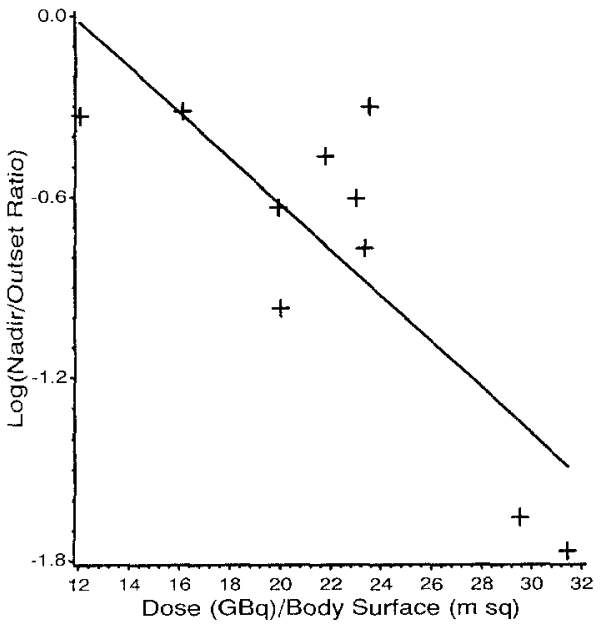

Fig. 3. $\log _{10}$-transformed platelet ratio (nadir/outset) versus dose of ${ }^{125} \mathrm{I}-\mathrm{MIBG}$ in $\mathrm{GBq} / \mathrm{m}^{2}$. The equation for the regression line is $\log _{10}($ ratio $)=0.89-0.0756 \times \mathrm{GBq} / \mathrm{m}^{2} ; r=-0.81, P=0.005$

similar to the others derived for this predictive index. A similar correlation was obtained by comparing the platelet ratio to $\mathrm{GBq} / \mathrm{m}^{2}$ to give the regression: ratio=2.62-0.244 GBq $/ \mathrm{m}^{2}$; then $r=-0.74, P=0.04$, and toxicity at a platelet ratio of 0.1 was predicted at 10.3 $\mathrm{GBq} / \mathrm{m}^{2}$ (Table 2).

Although data on absorbed radiation dose to blood were available for only four patients in this group, the correlation with the platelet ratio was $r=-0.84$.

\section{Treatment with ${ }^{125} I-M I B G$}

Unlike ${ }^{131}$ I-MIBG, none of the correlations between the various toxicity indices and cGy were statistically significant. However, the correlations between $\mathrm{GBq} / \mathrm{m}^{2}$ and $\mathrm{GBq} / \mathrm{kg}$ were statistically significant, and were fairly consistent across toxicity indices. They also tended to agree with the values produced for ${ }^{131} \mathrm{I}$-MIBG.

The highest correlation between predictive and toxic indices occurred when the $\log _{10}$-transformed platelet ratio was compared with the dose of ${ }^{125} \mathrm{I}-\mathrm{MIBG}$ measured in $\mathrm{GBq} / \mathrm{m}^{2}, r=-0.81 \quad(n=10, P=0.005)$ (Fig. 3). This toxicity index also gave a lower predicted toxic dose $\left(25.0 \mathrm{GBq} / \mathrm{m}^{2}\right.$ vs a mean of $28.1 \mathrm{GBq} / \mathrm{m}^{2}$ for the other three toxicity indices). The result was largely because the $\log _{10}$ transformation tended to emphasize the very low ratio values, and therefore produced a lower predicted toxic dose.

Since the effective half-life of ${ }^{125}$ I-MIBG is a major factor in calculating whole-body absorbed dose, the effective half-lives of the pretherapeutic or diagnostic dose (made with ${ }^{131}$ I-MIBG) are shown in Table 3 for patients treated with ${ }^{125} \mathrm{I}-\mathrm{MIBG}$. Four patients (\#2, \#5, $\# 8$, and \#10) had longer effective half-lives for the therapeutic dose than for the diagnostic dose, but, for all ten subjects, the mean effective half-life of the therapeutic dose $(25.5 \mathrm{~h})$ was less than that of the diagnostic
Table 3. Effective half -lives of whole-body diagnostic and therapeutic doses of ${ }^{125} \mathrm{I}-\mathrm{MIBG}$

\begin{tabular}{llll}
\hline Patient & Dx $T$ Eff $(\mathrm{h})$ & Rx $T$ Eff $(\mathrm{h})$ & $\mathrm{Rx} / \mathrm{Dx}$ \\
\hline 1 & 29.2 & 15.8 & 0.54 \\
$2^{\mathrm{a}}$ & 30.7 & 35.9 & 1.17 \\
3 & 27.5 & 14.6 & 0.53 \\
4 & 35.4 & 25.1 & 0.71 \\
5 & 23.4 & 28.3 & 1.21 \\
6 & 31.9 & 25.1 & 0.79 \\
7 & 27.9 & 20.8 & 0.75 \\
8 & 29.4 & 41.4 & 1.41 \\
$9^{\text {a }}$ & 64.8 & 26.6 & 0.41 \\
10 & 20.2 & 21.1 & 1.04 \\
Mean & 32.0 & 25.5 & 0.86 \\
SD & 12.3 & 8.3 & 0.33 \\
\hline
\end{tabular}

a One kidney

dose $(32.0 \mathrm{~h})$. The standard deviation of the therapeutic doses was smaller than that of the diagnostic doses, and, of particular interest, is that one of the patients who had a nephrectomy (patient \#9) exhibited a relatively long effective half-life $(64.8 \mathrm{~h})$ with the diagnostic dose, but the effective half-life of the therapeutic dose (26.6 h) was close to the mean for this group of patients. The other patient who had unilateral nephrectomy as part of the surgical resection of tumor (\#2) did not exhibit diagnostic or therapeutic half-life values that deviated substantially from the respective means of the group. The same was true for the three patients who had prior bone marrow transplantations (\#4, \#6, \#7).

The correlations of the absorbed radiation to blood with either of the toxicity indices gave low coefficients, the highest being $r=-0.17$.

\section{Discussion}

If ${ }^{131} \mathrm{I}-\mathrm{MIBG}$ and ${ }^{125} \mathrm{I}-\mathrm{MIBG}$ are to be effective in the treatment of neuroblastoma, the doses of these radiopharmaceuticals must be larger than have generally been given in the past. To achieve the greatest benefit, ${ }^{131}$ I-MIBG and ${ }^{125}$ I-MIBG must be given in the maximum tolerable doses. Yet, previous experience had not enabled reliable calculations of such doses. Although treatments with large, marrow-destroying doses of ${ }^{131} \mathrm{I}$ MIBG have been followed by bone marrow transplantation [22], this approach has not yet been widely applied nor validated. Assuming marrow transplantation is not part of the protocol, the limiting factor will be hematopoietic toxicity. Leukopenia followed treatments with ${ }^{131}$ I-MIBG and ${ }^{125}$ I-MIBG, but, with the exception of one patient whose entire marrow function was lost, the relative decline in leukocytes was less than that of platelets and did not pose an immediate threat to health. Platelet production has been the function most sensitive 
to the effects of radiation from the radiopharmaceuticals $[1,6,12,14]$, and, thus, the concentrations of circulating platelets have become the major index of toxicity. From our acquired data, we have demonstrated correlations between the level of thrombocytopenia and various indices of radiation dose so that bone marrow toxicity should be predictable with a reasonable degree of accuracy when either ${ }^{131}$ I-MIBG or ${ }^{125}$ I-MIBG is administered. However, our calculations were made on patients who had little or no bone marrow involvement by neuroblastoma, and they may not apply if there was an increment in marrow radiation from radiolabeled MIBG concentrated in proximate tumor cells.

Absorbed doses of radiation have been calculated for some individual organs for ${ }^{131} \mathrm{I}$-labeled monoclonal antibodies [23] and for ${ }^{111}$ In-labeled octreotide [24]. However, such measurements require additional assumptions and, although they seem to give reliable results for groups of people, the error for an individual patient may be substantial. In any case, our goal was to find a relatively simple and reproducible index of the earliest and most serious toxicity from radiolabeled MIBG, i.e., thrombocytopenia.

Although absorbed doses to bone marrow have been calculated by subtracting uptakes in tumor and liver from the whole-body retention of ${ }^{131}$ I-MIBG [25], the accuracy of such measurements is doubtful, especially since uptakes in organs and tumors are imprecise. Lacking any ready measurement of absorbed dose of radiation to the bone marrow, it was thought that whole-body dosimetry would give the highest level of correlation with the decline in circulating platelets. In the case of ${ }^{13}$ I-MIBG therapy, the cGy of whole-body radiation was predictive of thrombocytopenia, but with only a small advantage over the simpler and more easily attained index of $\mathrm{GBq} / \mathrm{m}^{2}$ of body surface area. For ${ }^{125} \mathrm{I}-$ MIBG treatments, absorbed doses of whole-body radiation had virtually no value in forecasting the changes in circulating platelets. Yet, $\mathrm{GBq} / \mathrm{kg}$ and $\mathrm{GBq} / \mathrm{m}^{2}$ each correlated with thrombocytopenia to give respectable $r$ values.

Why whole-body radiation doses should correlate with thrombocytopenia substantially less well than $\mathrm{GBq} / \mathrm{kg}$ or $\mathrm{GBq} / \mathrm{m}^{2}$ after treatments with ${ }^{125} \mathrm{I}-\mathrm{MIBG}$ is not clear. It is possible that the microdosimetry of ${ }^{125} \mathrm{I}$ MIBG in the bone marrow may vary from patient to patient, but using MIRD formalism, the only difference of consequence in deriving the indices was that doses in cGy take into account the effective half-life of the radiopharmaceuticals within the patients. The effective half-lives of the therapeutic doses differed from those used in the dosimetry calculations, but these discrepancies would degrade the correlations with toxicity only if the differences between pretherapeutic effective halflife and therapeutic half-life varied from patient to patient. This type of variation did not seem pronounced in patients treated with ${ }^{125} \mathrm{I}-\mathrm{MIBG}$, but may have played a role in the outcome. The hydration of the patients could have varied between the times of dosimetry and therapy and differed among patients. The consequence of different states of hydration may have been a more prolonged or briefer retention than predicted for ${ }^{125} \mathrm{I}$ MIBG in the tissues, including the bone marrow, of patients, and these variations may have contributed to errors in predicting toxicity by the use of the absorbed dose of radiation calculated from the diagnostic data. Thus, whole-body dosimetry incorporated more data than $\mathrm{GBq} / \mathrm{kg}$ and $\mathrm{GBq} / \mathrm{m}^{2}$ into an index of absorbed dose, but at the same time the added data introduced a greater variability that vitiated the use of cGy. Our whole-body dosimetry would probably have been more accurate if data had been acquired for one or two more days on the patients [25], but the limitations on patient expenses and the urgency for therapy dictated expediency in our protocol.

From the limited blood data in this study, calculations of radiation to blood appear to foretell the toxicity of ${ }^{131}$ I-MIBG as well. Following the injection of a diagnostic dose of ${ }^{131}$ I-MIBG, aspirates of bone marrow were obtained at the same time as blood specimens were drawn in three patients [14]; the respective concentrations of radioactivity were similar so that one might expect that blood dosimetry would reflect that in the marrow. However, blood dosimetry for ${ }^{125}$ I-MIBG had little or no correlation with the platelet levels following therapy with this agent. It is possible that radiation to the blood and body could increase consumption as well as decrease production of platelets, but there is no evidence that differing rates of loss of platelets play a major role in radiopharmaceutical treatments. There is still much to be learned about how ${ }^{125} \mathrm{I}-\mathrm{MIBG}$ imparts its destructive energy, including at the microdosimetry level.

The relative effects of ${ }^{125}$ I-MIBG and ${ }^{131} \mathrm{I}-\mathrm{MIBG}$ on normal tissues can be compared. To induce platelet declines to $10 \%$ of the pretherapeutic levels or to $20000 / \mu \mathrm{l}$, the doses of ${ }^{125} \mathrm{I}-\mathrm{MIBG}, 27.3 \mathrm{GBq} / \mathrm{m}^{2}$, were 2.6-fold higher than those of ${ }^{131} \mathrm{I}-\mathrm{MIBG}, 10.5 \mathrm{GBq} / \mathrm{m}^{2}$ (each value an average of four correlations). In three patients of our study, prior bone marrow transplantation did not seem to make for greater susceptibility to thrombocytopenia from radiopharmaceutical therapy.

In summary, we recommend that, in treatments with ${ }^{131} \mathrm{I}-\mathrm{MIBG}$, the predictive index $\mathrm{GBq} / \mathrm{m}^{2}$ be used with either the nadir or platelet ratio, either with or without the use of the $\log _{10}$ transformation. When ${ }^{125} \mathrm{I}-\mathrm{MIBG}$ is given as therapy, either $\mathrm{GBq} / \mathrm{kg}$ or $\mathrm{GBq} / \mathrm{m}^{2}$ will usefully predict the $\log _{10}$-transformed platelet ratio. The use of the $\mathrm{GBq} / \mathrm{m}^{2}$ predictive index against the $\log _{10}$-transformed platelet ratio produces acceptable results for both radiopharmaceutical treatments.

In addition, this type of analysis may prove useful for therapies employing other types of radiopharmaceuticals; empirical observations often make useful guides. 
Acknowledgements. The authors owe much to Mrs. Karen Grahl for help in preparing this manuscript. We also thank Dr. Larry Gruppen who helped in data analysis and figure preparation. The work was supported by grants FD-U-000558 from FDA, R01 CA 42768 from NIH, and M01 RR 00042 from NIH. The synthesis of ${ }^{131}$ I-MIBG and ${ }^{125}$ I-MIBG was carried out at the Phoenix Memorial Laboratory under the expert guidance of Clarke Hagen.

\section{References}

1. Hoefnagel CA, Voûte PA, De Kraker J, Valdés Olmos RA. $\left[{ }^{131} \mathrm{I}\right]$ Metaiodobenzylguanidine therapy after conventional therapy for neuroblastoma. J Nucl Biol Med 1991;35: 202206

2. Hör G, Maul FD, Kornhuber B, Schwabe D, Hesse J, Manegold KH, Baüm RP, Gerein V. Outcome of $\left[{ }^{131}\right.$ I]metaiodobenzylguanidine therapy of neuroblastoma: seven years after. $J$ Nucl Biol Med 1991;35: 207-215

3. Klingebiel T, Feine U, Treuner J, Reuland P, Handgretinger $R$, Neithammer $D$. Treatment of neuroblastoma with $\left[{ }^{131}\right.$ I] metaiodobenzylguanidine: long-term results in 25 patients. I Nucl Biol Med 1991;35: 216-219

4. Lumbroso J, Hartmann O, Schlumberger M. Therapeutic use of $\left.{ }^{131} \mathrm{I}\right]$ metaiodobenzylguanidine in neuroblastoma: a phase II study in 26 patients. I Nucl Biol Med 1991;35: 220-223

5. Claudiani F, Garaventa A, Bertolazzi L, Villavecchia GP, Cabria M, Scopinaro G, Bestagno M, Guerra P, Canevarollo N, Scielzo G, Lanino E, De Bernardi B. $\left[{ }^{131} \mathrm{I}\right]$ Metaiodobenzylguanidine therapy in advanced neuroblastoma. I Nucl Biol Med 1991;35: 224-227

6. Troncone L, Rufini V, Riccardi R, Lasorella A, Mastrangelo $R$. The use of $\left[{ }^{131} I\right]$ metaiodobenzylguanidine in the treatment of neuroblastoma after conventional therapy. $J$ Nucl Biol Med 1991;35: 232-236

7. Hutchinson RJ, Sisson JC, Miser JS, Zasadny KR, Normolle DP, Shulkin BL, Francis IR, Wieland DM, Shapiro B. Longterm results of $\left[{ }^{131} 1\right]$ metaiodobenzylguanidine treatment of refractory advanced neuroblastoma. J Nucl Biol Med 1991;35: $237-240$

8. Castellani MR, Rottoli L, Maffioli L, Massimino M, Gasparini M, Buraggi GL. Experience with palliative $\left[{ }^{131} I\right]-$ metaiodobenzylguanidine therapy in advanced neuroblastoma. J Nucl Biol Med 1991;35: 241-243

9. Matthay KK, Huberty JP, Hattner RS, Ablin AR, Engelstad BL, Zoger S, Hasegawa BH, Price D. Efficacy and safety of $\left[{ }^{131} 1\right]$ metaiodobenzylguanidine therapy for patients with refractory neuroblastoma. I Nucl Biol Med 1991;35: 244-247

10. Hoefnagel CA, De Kraker J, Voûte PA, Valdés Olmos RA. Preoperative $\left[{ }^{131} I\right]$ metaiodobenzylguanidine therapy of neuroblastoma at diagnosis ("MIBG de novo"). J NuCl Biol Med 1991;35: 248-251

11. Mastrangelo R, Lasorella A, Troncone L, Rufini V, Iavarone A, Riccardi R. $\left[{ }^{131} \mathrm{I}\right]$ Metaiodobenzylguanidine in neuroblastoma patients at diagnosis. $J$ Nucl Biol Med 1991;35: 252-254
12. Sisson JC, Hutchinson RJ, Shapiro B, Zasadny KR, Normolle D, Wieland DM, Wahl RL, Singer DA, Mallette SA, Mudgett EE. Iodine-125-MIBG to treat neuroblastoma: preliminary report. J Nucl Med 1990;31: 1479-1485

13. Sisson JC, Shapiro B, Hutchinson RJ, Zasadny KR, Mallette S, Mudgett E, Wieland DM. Treatment of neuroblastoma with $\left[{ }^{125}\right.$ I]metaiodobenzylguanidine. I NuCl Biol Med 1991;35: 255-259

14. Sisson JC, Hutchinson RJ, Carey JE, Shapiro B, Johnson JW, Mallette SA, Wieland DM. Toxicity from treatment of neuroblastoma with ${ }^{131} \mathrm{I}$-metaiodobenzylguanidine. Eur J Nucl Med 1988;14: 337--340

15. Hutchinson RJ, Sisson JC, Shapiro B, Miser JS, Normolle D, Shulkin BL, Francis IR, Zasadny KR, Carey JE, Johnson JW, Mallette SA, Mudgette B. 131-I-Metaiodobenzylguanidine treatment in patients with refractory advanced neuroblastoma. Am J Clin Oncol 1992;15: 226-232

16. Mangner TJ, Wu JL, Wieland DM. Solid phase exchange radioiodination of aryl iodides. Facilitation by amonium sulfate. J Org Chem 1982;47: 1484-1488

17. Sisson JC, Shapiro B, Beierwaltes WH. Radiopharmaceutical treatment of malignant pheochromocytoma. $J$ Nucl Med 1984;24: 197-206

18. Lovinger R, Berman M. A revised schema for calculating the absorbed dose from biologically distributed radionuclides. MIRD Pamphlet No. 1, Revised. Society of Nuclear Medicine; 1976

19. ICRU (1979). Methods of assessment of absorbed dose in clinical use of radionuclides. ICRU Report 32. Washington: International Commission on Radiation Units and Measurements, 1979

20. Brownell WH, Ellett WH, Reddy AR. Absorbed fractions for photon dosimetry. MIRD Pamphlet No. 3. New York: The Society of Nuclear Medicine; 1968

21. Fleiss J. Statistical methods for rates and proportions, 2nd $e d n$. New York: John Wiley \& Sons, 1981

22. Corbett R, Pinkerton R, Tait D, Meller S. ${ }^{131}$ I]Metaiodobenzylguanidine and high-dose chemotherapy with bone marrow rescue in advanced neuroblastoma. $J \mathrm{Nucl}$ Biol Med 1991;35: 228-231

23. Sgouras G, Graham MC, Divgi CR, Larson SM, Scheinberg DA. Modeling and dosimetry of monoclonal antibody M195 (anti-CD33) in acute myelogenous leukemia. $J \mathrm{NuCl} \mathrm{Med}$ 1993;34: 422-430

24. Krenning EP, Bakker WH, Kooij PPM, Breeman WAP, Oei HY, de Jong M, Reubi JC, Visser TJ, Bruns C, Kwekkeboom DJ, Reijs AEM, van Hagen PM, Koper JW, Lamberts SWJ. Somatostatin receptor scintigraphy with indium-111-DTPA-Dphe-1-octreotide in man: metabolism, dosimetry and comparison with iodine-123-tyr-3-octreotide. J NuCl Med 1992;33: $652-658$

25. Lashford LS, Moyes J, Ott R, Fielding S, Babich J, Mellors S, Gordon I, Evans K, Kemshead JT. The biodistribution and pharmacokinetics of metaiodobenzylguanidine in childhood neuroblastoma. Eur J Nucl Med 1988;13: 574-577 